\title{
Germanica
}

\section{Die Aktualisierung des Bibeltextes in Alfred Döblins Montageroman Berlin Alexanderplatz. Die Geschichte vom Franz Biberkopf}

Die Aktualisierung des Bibeltextes in A. Doblins Montageroman Berlin

Alexanderplatz. L'actualisation du texte biblique dans le roman Berlin

Alexanderplatz d'Alfred Döblin

\section{Monique Boussart}

\section{(2) OpenEdition}

\section{Journals}

Édition électronique

URL : http://journals.openedition.org/germanica/2072

DOI : 10.4000/germanica.2072

ISSN : 2107-0784

\section{Éditeur}

Université de Lille

\section{Édition imprimée}

Date de publication : 31 décembre 2002

Pagination : 99-111

ISBN : 9782913857100

ISSN : 0984-2632

\section{Référence électronique}

Monique Boussart, «Die Aktualisierung des Bibeltextes in Alfred Döblins Montageroman Berlin

Alexanderplatz. Die Geschichte vom Franz Biberkopf », Germanica [Online], 31 | 2002, Online erschienen am: 19 März 2013, abgerufen am 06 Oktober 2020. URL : http://journals.openedition.org/germanica/ 2072 ; DOI : https://doi.org/10.4000/germanica.2072

Ce document a été généré automatiquement le 6 octobre 2020.

(c) Tous droits réservés 


\title{
Die Aktualisierung des Bibeltextes in Alfred Döblins Montageroman Berlin Alexanderplatz. Die Geschichte vom Franz Biberkopf
}

\author{
Die Aktualisierung des Bibeltextes in A. Doblins Montageroman Berlin \\ Alexanderplatz. L'actualisation du texte biblique dans le roman Berlin \\ Alexanderplatz d'Alfred Döblin
}

Monique Boussart

1 Die Bibel ist zweifellos eine der grundlegenden Quellen, die Künstler und Dichter zu eigenen Schöpfungen angeregt haben. Wie und wozu ein Romancier der späten zwanziger Jahre, der noch der expressionistischen Utopie vom «neuen Menschen » nachhängt und zugleich dem sozialpolitischen Anliegen der Neuen Sachlichkeit zugetan ist, diesen kanonischen Text in seinem Großstadtroman auswertet, diesen Fragen möchte ich hier nachgehen.

2 Bekannt ist, dass Döblin sich in seinem 1929 erschienenen Werk überwiegend auf das Alte Testament bezieht - von den allerersten Seiten bis zum Schluss -, nämlich auf die Genesis, das Buch Hiob, die Klagen Jeremias und des Predigers Salomo ${ }^{1}$. Außer der berühmten Entlehnung des Motivs der Hure Babylon aus der Offenbarung Johannis sind nur kürzere Zitate aus dem Neuen Testament in seinen Text einmontiert ${ }^{2}$, die sozusagen den Bildungsstand des Protagonisten, des kleinen Mannes widerspiegeln. Was ihren Umfang anbetrifft, reichen die biblischen Bezüge von recht vagen Reminiszenzen, Hinweisen und Anspielungen ${ }^{3}$ bis zu wörtlichen Zitaten, längeren Paraphrasen und Nacherzählungen, sogar Um- und Weiterdichtungen. Wo auch immer sie auftreten, stets werden sie unvermittelt und kommentarlos eingefügt. 


\section{Der Einbezug des biblischen Textes im Rahmen der Erneuerung des Romans}

Döblins Inanspruchnahme der Lutherschen Bibel hat nichts Verwunderliches an sich, sie steht völlig im Einklang mit seiner Poetik. Schon in seinen frühesten Aufsätzen zur Literatur tritt er als dezidierter Gegner des konventionellen psychologisierenden Handlungsromans auf und verlangt nachdrücklich eine Neubelebung der Gattung. Der Rekurs auf biblische Parabeln und Sprüche ist mit seiner Bevorzugung einer assoziativen, auf Analogie beruhenden Schreibtechnik verknüpft, die eher zur Suggestion als zur Explikation greift und dem Leser eine aktivere Rolle zuweist. Er entspricht den Richtlinien, die der Schriftsteller in seinem ebenfalls 1929 erschienenen Essay « Der Bau des epischen Werks » für den Wandel des Romans in ein modernes Epos festlegt. Das Hauptcharakteristikum des epischen Berichts, "das Exemplarische des Vorgangs und der Figuren $»^{4}$, findet er im Alten und im Neuen Testament wieder sowie die « überreale Sphäre », die lyrischen und dramatischen Momente, denen der epische Autor nach Döblin ebenfalls einen Platz gewähren sollte. Vielsagend ist, dass der Essayist im Abschnitt über Sprachstile und Stilebenen ausdrücklich auf die Luthersche Bibelübersetzung hinweist und Folgendes dabei betont :

[...] und wer auf diese Ebene tritt, muss wissen, wohin er tritt, muss wissen, wohin ihn diese Sprache weitertreibt und dass das Ergreifen auch nur von einigen Sätzen sowohl zu anderen Sätzen derselben Ebene, aber auch zu Gedanken und Vorstellungen dieser Ebene drängt. Mit anderen Worten : jedem Sprachstil wohnt eine Produktivkraft und ein Zwangscharakter inne, und zwar ein formaler und ein ideeller. (S.130 f).

Inwieweit Döblin tatsächlich für diesen formalen und ideellen «Zwangscharakter » aufnahmebereit war, werde ich jetzt zu beleuchten versuchen.

In seiner Behandlung der biblischen Vorlage sind die Hauptkennzeichen der intertextuellen Praxis der Hauptvertreter der Moderne, wie etwa Joyce oder Th. Mann, wiederzuerkennen : die Mehrschichtigkeit, die funktionale bzw. strukturierende Rolle der eingegliederten Elemente, das spielerische Moment sowie die ironische und autoreflexive Brechung, die zur Potenzierung des dichterischen Reizes beiträgt.

\section{Zur funktionalen und strukturierenden Rolle der eingeblendeten Bibelstellen}

6 Die Bibel ist dem Autor weit mehr als ein bloßer Fundus von Bildern, die zur Textausschmückung dienen. Werner Stauffacher bezeichnet sogar den «Bibelhorizont» als "umfassende Hintergrundstruktur» des Romans 5 . Die alttestamentlichen Parabeln und Sprüche finden sich an Angelpunkten der Geschichte ; in den jeweiligen Handlungskontext genau eingepasst, führen oder rahmen sie die Schlüsselszenen ein. Außerdem werden sie oft zu Leitmotiven, so verbinden sie einzelne Erzählsequenzen miteinander, lassen sich als Vorausdeutungen oder Reprisen erkennen und gewährleisten auf diese Weise die Kohärenz des äußerst dichten und mosaikartig fragmentierten Romanwerks.

7 Die Sinndeutung erscheint als eine der Hauptfunktionen der Bibelbezüge : sie enthüllen den eigentlichen Stellenwert der Hauptfiguren und ihrer Erlebnisse, somit erweisen sie 
sich als wesentliche Orientierungshilfe für den Leser. Unter den vielen heterogenen Montageingredienzien hat die biblische Komponente, wie sich bald herausstellt, eine spezifische Aufgabe. Während die Werbesprüche, die Schlager-, Lieder-, Zeitungs- und Verordnungsauszüge den örtlichen und zeitlichen Hintergrund veranschaulichen, den Berliner Osten und die sozialpolitischen Spannungen der späten zwanziger Jahre heraufbeschwören, stehen die alttestamentlichen Passagen in enger Verbindung mit der inneren Entwicklung des Protagonisten Franz Biberkopf und mit den ethischen Fragen, die sie aufwirft ${ }^{6}$. Der hier als Moralist auftretende Romancier setzt die Bibelstellen ein, um eine Lehre zu vermitteln, ohne aber auf den erzählerischen Duktus verzichten und sich auf das Diskursive beschränken zu müssen, ein Verfahren, das ihm auch die chassidischen Erzählungen vorführten, die er während seiner Reise nach Polen im Jahre 1924 kennenlernte. In diesem Sinne bekräftigt der Bibeltext den didaktischen Inhalt der bänkelsängerischen Vorreden zu den einzelnen Büchern des Romans, freilich in einer ganz anderen Tonlage. Dies sei an der Verarbeitung von zwei alttestamentlichen Exempeln veranschaulicht, die einen zentralen Platz in Berlin Alexanderplatz einnehmen, die beide auf Prüfung und Erkenntnis angelegt sind und offensichtlich als Parallelen und Vorausdeutungen des Schicksals der Hauptfigur konzipiert wurden.

8 So wird die Hiob-Erzählung zwischen die beiden Schlachthof-Abschnitte, jene metaphorischen Darstellungen von Biberkopfs und Miezes Auslieferung an die Gewalt, eingefügt (S.143 ff). Die Du-Form verbindet schon rein formal den Bericht des Erzählers über Biberkopfs Flucht aus der Wirklichkeit ins Saufen mit der Hiob-Episode : Dieser Bericht präsentiert sich nämlich als mahnende Rede an die Hauptfigur und findet eine Art Fortsetzung in dem darauffolgenden Dialog zwischen einer rätselhaften Stimme und dem Geprüften. Die Stimme, die auf den ersten Blick noch als diejenige des Erzählers identifiziert werden könnte, sich dann eher, dem biblischen Kontext entsprechend, als die Satans erkennen lässt, rekapituliert Hiobs Verluste und konzentriert sich auf die Frage, woran Hiob am meisten gelitten hat. Darauf kann dieser nur jammernd, flehend und protestierend antworten. Obwohl Döblin den biblischen Topos der Ertönung einer geheimnisvollen Stimme und des Zwiegesprächs mit der numinosen Erscheinung übernimmt, weicht seine Version doch erheblich vom Bibeltext ab. Der Satan, der im Alten Testament bloß im Prolog auftritt - « der Herr » erlaubt ihm, Hiobs Gottergebenheit auf die Probe zu stellen - spielt hier die Hauptrolle. Der Dialog ist im Original einer zwischen Hiob und seinen Freunden, an die er seine Klage richtet. Beim Berliner Schriftsteller kommen die Freunde überhaupt nicht vor, was ihn nicht daran hindert, einzelne Motive und Wendungen aus dem Gespräch mit ihnen wiederaufzunehmen (Buch Hiob, K. 9, 10, 30). Der Blickwinkel verengt sich, dadurch gewinnt die Mühseligkeit der Lehreerteilung und der Bewusstwerdung an Gewicht und Wirksamkeit. Was den Döblinschen und den biblischen Hiob miteinander verbindet, ist die Klage über die Ungerechtigkeit ihres Geschicks. Im Roman aber wird hauptsächlich herausgearbeitet, dass Hiobs eigene Schwächen, sein Aufbegehren und sein Selbstmitleid, für sein Unglück verantwortlich sind. Für Döblin ist Hiob eben nicht der Gerechte und unschuldig Geprüfte. Er fokussiert das Ganze auf seinen Mangel an Einsicht und seine Ichverkrampfung. Die häufige Wiederholung des Verbs «nicht wollen », des « nein » und die Überschrift « [...] es liegt an dir, Hiob, du willst nicht » (S. 143) lassen keinen Zweifel darüber zu. Die ganze Distanz zwischen dem Roman und der Vorlage offenbart sich im Abschluss der Geschichte. Zwar wird hier wie dort Hiob erst geheilt, als er auf seine Rebellion verzichtet. In der Bibel aber ist Hiobs Ergebung in 
Gottes Willen eine Reaktion auf die Rede des Herrn, die der Ohnmacht des Menschen die eigene Allmacht entgegenstellt. Nichts dergleichen bei dem deutschen Romancier, der nur die Anhaltung zur inneren Wandlung und zum Aufgeben der Flucht vor der Wirklichkeit herausstellt. Diese Säkularisierung ${ }^{7}$ spiegelt sich im Stil : der feierliche Ernst des Alten Testaments ist einer einfacheren, farbigen Sprache und einem schnelleren Tempo gewichen :

Hiob, du liegst im Kohlgarten, an der Hundehütte, grade so weit weg, dass dich der Wachhund nicht beißen kann. Du hörst das Knirschen seiner Zähne. Der Hund bellt, wenn sich nur ein Schritt naht. Wenn du dich umdrehst, dich aufrichten willst, knurrt er, schießt vor, zerrt an seiner Kette, springt hoch, geifert und schnappt. Hiob, das ist der Palast, und das sind die Gärten und die Felder, die du selbst einmal besessen hast. [...] Hiob, jetzt hast du alles verloren ${ }^{8}$.

Ein Pendant zu dieser Hiobdeutung bildet im 6. Buch die Episode von Isaaks Opferung (S. $284 \mathrm{ff} ; 1$. Mos. 22. 3 ff). Auch diesmal sind die Verschiebung des thematischen Schwerpunkts und die formale Umgestaltung kennzeichnend. Die Abraham-IsaakGeschichte wird in den Abschnitt über die Hilflosigkeit des zum Krüppel gewordenen und zur Arbeitslosigkeit verurteilten Biberkopf eingeschoben. Von der alttestamentlichen Erzählung übernimmt der Autor allein das Mittelstück. Er erweitert den im Original sehr kurzen Dialog zwischen Abraham und seinem Sohn, das Zwiegespräch zwischen Abraham und Gott nimmt dagegen nur einen sehr geringen Raum ein. In der Bibel werden die Gefühle von Abraham und Isaak ausgespart, im Roman stehen sie im Vordergrund. Eine deutliche Humanisierung und Verweltlichung des Vorgangs lässt sich also beobachten. In diesem Zusammenhang kann das von Döblin breit ausgeführte Motiv vom Gang zum Opferungsberg, «bergauf, bergab », als Metapher für den Lebensgang des Menschen mit seinen vielen Wechselfällen ausgelegt werden. Wo es im 1. Buch Mose um den unbedingten Gehorsam gegenüber Gott geht, dreht es sich im Roman um die Furcht vor der Selbsthingabe. Döblin zentriert die Erzählung um die Bemühungen des Vaters, eigentlich das Sprachrohr des lehrenden Autors, den Sohn zur Einwilligung in das Opfer, in den Tod zu bewegen. Dies steigert die dramatische Spannung des Textes, der durch die Insistenz von Wendungen wie " fürcht dich nicht », « tu es gern », « wolle es doch » geprägt ist. Das Ende der Episode bleibt der Tradition treu : erst als Isaak zum Äußersten bereit ist, erst dann erfolgt die Rettung.

10 Auf stilistischer Ebene ersetzt eine höchst pathetische Sprache die Sachlichkeit und trockene Härte des Alten Testaments 9 . Aus dem Abraham-und-Isaak-Exempel macht Döblin eine im überstürzten Tempo erzählte Schauerballade mit dramatischem Dialog, lyrischen Momenten und Überraschungseffekten. Die stark rhythmisierte Prosa, die zahlreichen Klang- und Wortwiederholungen und das als Refrain wiederkehrende «bergauf, bergab, die langen Täler« sind in dieser Hinsicht charakteristisch. Der biblische Stoff gewinnt eine neue Lebendigkeit ; sogar die Landschaft, so gut wie nicht vorhanden in der Genesis, wird sinnfällig angedeutet :

Und da ist ein Gebirge und der alte Mann steht auf und sagt zu seinem Sohn : Komm mit. Komm mit, sagt der alte Mann zu seinem Sohn und geht und der Sohn geht mit, geht hinterdrein ins Gebirge hinein, hinauf, hinunter, Berge, Täler. Wie lange gehts noch, Vater? Das weiß ich nicht, wir gehen bergauf, bergunter, ins Gebirge, komm nur mit. [...] Bergauf, bergab, Täler, es ist ein langer Weg [...] (S.284).

11 Der Dichter lässt seiner Phantasie freien Lauf und schlägt einen volkstümlichen, naiven, ja spielerischen Ton an, wie aus dem vierzehnmal wiederholten Hallelujah am Schluss herauszuhören ist: «Hallelujah, [...] Hallelujah, luja, luja, lujah, hallelujah, 
lujah, hallelujah. » (S.285). Auf Biberkopfs Werdegang bezogen, - erst als Biberkopf sich nach vielen Irrungen dem Tode stellt, wird er zum neuen Menschen -, nimmt sich dieses Hallelujah als kritisch-spöttisches Lächeln des Erzählers und Mentors aus.

Die ethische Haltung, die aus Döblins Varianten der biblischen Erzählungen, herauszulesen ist, der Verzicht auf die Selbstbewahrung und die Annahme des Leidens, gehört, genau wie die Spannung zwischen Hochmut und Demut, zum eigentlichen Kern seiner Weltanschauung, sie zeichnet sich ja schon in Die drei Sprünge des Wang-Lun (1915) ab. In der Schlusspartie von Berlin Alexanderplatz wird sie nochmals durch eine kurze litaneiartige Paraphrase der Geschichte vom Märtyrertod der Makkabäer (S. 444 ; nach 2. Makk. 7) hervorgehoben. Im Grunde entspricht Döblins Verfahren genau Julia Kristevas Definition der Intertextualität als « Transposition » eines Zeichensystems in ein anderes : Der fremde Text findet Eingang in ein bestimmtes Netzwerk mit seinen spezifischen Gesetzen.

\section{Die ästhetische Dimension. Kunst der Variation und kompositorische Virtuosität}

13 Es wäre verfehlt, Döblins Rückgriff auf die Heilige Schrift auf seine didaktische und erbauliche Intention zu beschränken. Selbst in diesem Bereich bricht er mit den Konventionen: die ironische Distanzierung von den eigenen Figuren und die raffinierten Variationen in Struktur und Sprache bezeugen es. So tragen die biblischen Einschübe auch wesentlich zur Steigerung des literarischen Reizes bei. In dieser Beziehung sind die Bearbeitung und die Integration der Entlehnungen aus dem 1. Buch Mose, insbesondere des Adam-und-Eva-Motivs in den ersten Teilen von Berlin Alexanderplatz, aufschlussreich. Zuerst begleitet die Evokation von Adams und Evas Glück im Paradies den Entschluss Biberkopfs, der soeben aus dem Gefängnis entlassen wurde, fortan anständig zu bleiben :

\footnotetext{
Es lebten einmal im Paradies zwei Menschen, Adam und Eva. Sie waren vom Herrn hergesetzt, der auch Tiere und Pflanzen und Himmel und Erde gemacht hatte. Und das Paradies war der herrliche Garten Eden. Blumen und Bäume wuchsen hier, Tiere spielten rum, keiner quälte den andern. Die Sonne ging auf und unter, der Mond tat dasselbe, das war eine einzige Freude den ganzen Tag im Paradies. So wollen wir fröhlich beginnen. Wir wollen singen und uns bewegen : Mit den Händen klapp, klapp, klapp, mit den Füßchen trapp, trapp, trapp, einmal hin, einmal her, ringsherum, es ist nicht schwer. (S. 49 ; nach 1. Mos. $2.8 \mathrm{f}$ ).
}

Der von der Vorlage stark abweichende Ton, der sich dem der Kinderliteratur annähert, was die Zeilen aus einem Couplet von « Hänsel und Gretel » noch verstärken, lässt, wenn auch spielerisch, die kritische Einstellung des Erzählers zur Hauptfigur durchschimmern : die Naivität ihres Selbstvertrauens wird indirekt entlarvt. Die oben angeführten Sätze klingen mehrere Seiten weiter nach. Diesmal verraten sie, nicht ohne ironischen Beiklang, die Sehnsucht des durch den Dschungel der Großstadt irrenden Biberkopf (S. 95).

Im weiteren Verlauf der Geschichte steht eher die Vertreibung aus dem Paradies im Vordergrund, und das sobald der Protagonist mit der Arbeitslosigkeit konfrontiert wird (S. 82 ;1. Mos. 3. 24) ; beim Erlebnis des Betrugs und Verrats durch den Freund Lüders beherrscht das Motiv der Versuchung und des Sündenfalls das Blickfeld. Es wird noch 
eindringlicher, als der entmutigte Biberkopf sich wieder in verbrecherische Machenschaften mitreißen lässt :

Die Schlange war vom Baum geraschelt. Verflucht sollst du sein mit allem Vieh, auf dem Bauch sollst du kriechen, Staub fressen zeitlebens. Feindschaft soll gesetzt sein zwischen dir und deinem Weibe. Mit Schmerzen sollst du gebären, Eva. Adam, verflucht soll der Erdboden sein um deinetwillen, Dornen und Disteln sollen drauf wachsen, Kraut des Feldes sollst du essen. (S.134 ; nach 1. Mos. 3. 14-18. Siehe auch S. 150).

Döblin übernimmt kurze Wendungen aus dem Original, er hält sich an den durch Parataxen und Wiederholungen gekennzeichneten Sprachrhythmus, aber er nimmt Raffungen zur Intensivierung der Ausdruckskraft vor. Eine solche Intensivierung erreicht er auch durch das gegenteilige Verfahren, nämlich durch das obsessive Wiederholen des Zitats. Das gilt unter anderem für die Worte « des Herrn», die den Abschluss dieses Motivkomplexes bilden : «Von Erde bist du gekommen, zu Erde sollst du wieder werden» (1. Mos. 3. 19). Dreimal erklingen sie (S.119), den Tiefpunkt von Biberkopfs Verzweiflung andeutend, und kehren nochmals am Anfang des 5. Buchs wieder (S.167).

Die eigenwillige Verfremdung der Vorlage, ohne aber in die Parodie zu verfallen, dokumentiert H. Meyers Bemerkungen über die Kunst des Zitierens. Er stellt in der Tat die eigenartige Spannung zwischen Assimilation und Dissimilation heraus : Das Zitat verbinde sich eng mit seiner neuen Umgebung, aber hebe sich zugleich von ihr $\mathrm{ab}^{10}$.

\section{Prophetische Sprüche als Reflexionspausen und Kommentare des Erzählers}

18 Im «Bau des epischen Werks» erkannte Döblin dem Autor das Recht des «Mitsprechens » zu, ein Recht, von dem er in Berlin Alexanderplatz reichlich Gebrauch macht, aber oft indirekt, wie schon aus seinen kritischen Hinweisen auf die eigenen Gestalten hervorgeht. Die Drohworte oder Klagen Jeremias und des Predigers Salomo, an deren Wortlaut er sich ziemlich genau hält, stellen sich als universalisierende Bemerkungen des Erzählers zu den Figuren oder allgemeiner zur menschlichen Hinfälligkeit und Ohnmacht heraus. Die Biberkopf-Handlung erscheint somit als Offenbarung der Conditio humana.

19 Am Anfang des Romans wird im Zusammenhang mit dem tief verstörten, soeben aus dem Gefängnis entlassenen Biberkopf auf Jeremias Spruch Bezug genommen :

Sprach Jeremia, wir wollen Babylon heilen, aber es ließ sich nicht heilen. Verlasst es, wir wollen jeglicher nach seinem Lande ziehen. Das Schwert komme über die Kaldäer, über die Bewohner Babylons. (S. 21 ; nach Je. 50. 16, 35).

Das frühe Aufklingen des Babylon-Themas, das in der zweiten Hälfte und am Ende des «Epos" eine entscheidende Rolle spielt, stellt die auf diese besondere Weise eingerahmte Biberkopf-Fabel von vornherein in eine bestimmte Perspektive hinein. Ähnlich verhält es sich mit den am Ende des 5. Buchs eingefügten Jeremia-Versen (17. 5-9), die die Beziehung zwischen dem Protagonisten und Reinhold, dessen Widersacher und bösem Geist, ins wahre Licht rücken :

Verflucht ist der Mann, spricht Jeremia, der sich auf Menschen verlässt, der das Fleisch zu seiner Stütze macht und dessen Herz von Gott abfällt. Er gleicht einem Verlassenen in der Steppe und gewahrt es nicht, wenn Gutes kommt. Er weilt im Dürren, in der Wüste, auf salzigem Boden, der nicht bewohnt ist. Gesegnet, 
gesegnet, gesegnet ist der Mann, der auf Gott vertraut und dessen Zuversicht der Herr ist. Er gleicht einem Baum, der am Wasser gepflanzt ist und seine Wurzeln in den Bach erstreckt. [...] Das Herz ist trügerisch über alles und ist verderbt ; wer mag es kennen? (S. 197 f. Siehe auch S.211 f).

21 Statt die psychische Verfassung, die Angst und Trauer seiner zur Krüppelexistenz verdammten Hauptfigur umständlich zu kommentieren, greift der Autor zu der klagenden Rede des Propheten :

[...] ich muss auf den Bergen weinen und heulen und bei den Herden in der Wüste klagen, denn sie sind so verheert, dass niemand da wandelt, es ist beides, Vögel des Himmels und das Vieh, alles weg [...] Ich will Jerusalem zum Steinhaufen und zur Wohnung der Schakale machen und will die Städte Judas wüste machen, dass niemand drinnen wohnen soll. (S. 270 ; nach Jer. 9. 9-10).

Bemerkenswert ist, dass die feierlichen Aussagen unvermittelt in die Unterhaltung zwischen einem alten Arbeiter und Biberkopf eingeschaltet werden, die Hohe Sprache und die poetischen Vergleiche des Alten Testaments stehen im schroffsten Gegensatz zur nüchternen und saloppen Umgangssprache des Berliners.

Dies gilt auch für die elegischen Passagen, die Döblin dem Prediger Salomo entlehnt. So lautet die Überschrift der sachlich einsetzenden Schlachthof-Episode : "Denn es geht dem Menschen wie dem Vieh; wie dies stirbt, so stirbt er auch » (S 136; nach Pred. 3. 19). Sie wird erst im Titel des zweiten Schlachthof-Abschnitts vervollständigt, « Und haben all einerlei Odem, und der Mensch hat nichts mehr denn das Vieh » (S.146 ; Pred. 3. 19), und macht die metaphorische Bedeutung der Tierschlachtung vollends klar.

Die prophetischen Sätze werden immer drängender, je mehr sich Biberkopf seinem Zusammenbruch nähert ${ }^{11}$, sie unterstreichen die sich zuspitzende Lage der Romangestalten. Vom 7. Buch an, das von der Ermordung Miezes, Biberkopfs Geliebten und einziger Stütze, erzählt, durchziehen die Worte des Predigers über die Vergänglichkeit leitmotivisch den Text; sie tragen zur Verstärkung der dramatischen Intensität entscheidend bei und verleihen diesem Teil des Werks seinen spezifischen Ton. Zum erstenmal erklingt das Zitat bei der Beschreibung von Reinholds Verführungsversuchen während eines Waldspaziergangs mit Mieze :
Ein Jegliches, ein Jegliches hat seine Zeit und alles Vornehmen unter dem Himmel hat seine Stunde, ein Jegliches hat sein Jahr, geboren werden und sterben, pflanzen und ausrotten, das gepflanzt ist, ein Jegliches, Jegliches hat seine Zeit, würgen und heilen, brechen und bauen, suchen und verlieren, seine Zeit, behalten und wegwerfen seine Zeit, zerreißen und zunähen, schweigen und reden. Ein Jegliches hat seine Zeit. Darum merkt ich, dass nichts Besseres ist, als fröhlich sein. Besseres als fröhlich sein. Fröhlich sein, lasst uns fröhlich sein. Es ist nichts Besseres unter der Sonne als lachen und fröhlich sein (S. 346 ; Pred. 3. 1-7, 12).

Auch diesmal lassen sich vielsagende Abwandlungen des Hypotextes feststellen. Döblin hält sich an die Motivik des Predigers, an die stereotypischen Wendungen und den litaneiartigen Satzrhythmus mit der charakteristischen polysyndetischen Struktur und der Wiederkehr der Gegensatzpaare. Er intensiviert jedoch die rhythmische Eindringlichkeit, indem er die Wortgruppen zu binären und alliterativen Verbindungen ( "brechen/bauen », «zerreißen/zunähen ») verkürzt. Auffällig ist die Wiederholung der abschließenden Empfehlung « fröhlich sein », die im Alten Testament als Freude an Gottes Gaben trotz der Eitelkeit des Diesseits verstanden wird, hier aber im Zusammenhang mit dem erotischen Spiel zwischen Reinhold und der ahnungslosen Mieze steht. Im weiteren Verlauf der Ereignisse nimmt der Vers «Jegliches, ein Jegliches hat seine Zeit» eine drohendere Bedeutung an, er unterstreicht jetzt die 
Unabwendbarkeit von Miezes Schicksal, Bruchstücke des oben angeführten Zitats begleiten ihre Passion bis zu ihrer Erwürgung im Walde. Darauf folgt die verzweifelte Aussage des Predigers, der kein anderer ist als der alles überblickende Autor :

Und ich wandte mich und sah an alles Unrecht, das geschah unter der Sonne, und siehe da, es waren Tränen derer, so Unrecht litten und hatten keinen Tröster, und die ihnen unrecht taten, waren zu mächtig. Da lobte ich die Toten, die schon gestorben waren. [...] Die Toten lobte ich, die unter den Bäumen liegen, die schlafen (S. 363 ; nach Pred. 4).

Unüberhörbar dabei ist, am Ende des Passus, der deutliche Bezug auf Miezes Tod ${ }^{12}$.

\section{Das biblische Wort, ein Mittel zur symbolischen Überhöhung}

27 Der Rückgriff auf das Alte Testament ermöglicht es dem Dichter, das Erzählte in eine umfassendere Perspektive hineinzustellen und der Biberkopf-Fabel eine metaphysische Bedeutung zu verleihen. Noch deutlicher ist dies bei der Einblendung des Motivs der Hure Babylon aus der Offenbarung Johannis.

Nicht von ungefähr setzt Döblin es zum erstenmal im 6. Buch ein, d.h. an strategischer Stelle, etwa in der Mitte des Romans, da der müde "Eroberer» zum Hehler und Zuhälter geworden ist; wie der Engel der Apokalypse wendet sich der Erzähler an den Protagonisten :

Und nun komm her, du, komm, ich will dir etwas zeigen. Die große Hure, die Hure Babylon, die da am Wasser sitzt. Und du siehst ein Weib sitzen auf einem scharlachfarbenen Tier. Das Weib ist voll Namen der Lästerung und hat 7 Häupter und 10 Hörner. [...] Und an ihrer Stirn ist geschrieben ein Name, ein Geheimnis : die große Babylon, die Mutter aller Greuel auf Erden. Das Weib hat vom Blut aller Heiligen getrunken. Das Weib ist trunken vom Blut der Heiligen. (S. 237 ; nach Offb. 17. 1, 3-6).

Von nun an durchziehen diese Zeilen, als Warnung, die letzten Bücher des "Epos", wobei Döblin kennzeichnenderweise das biblische "ich sah» $z u$ "du siehst» umwandelt.

Die Heraufbeschwörung der mythischen Figur kündigt die verhängnisvolle Konfrontation des Einzelnen mit dem Verbrechen und der Gewalt an. Sie wird mehrmals, verkürzt und leicht variiert, im Zusammenhang mit Reinhold (S. 253, 291) und nach Miezes Ermordung (S. 380) in den Text eingeflochten; als retardierendes und Spannung erhöhendes Moment klingt sie noch ein paar Mal nach in der letzten Phase von Biberkopfs Leidensweg. Der Romanschluss zeigt dann, dass Döblin der apokalyptischen Gestalt sein eigenes Gepräge verleiht, indem er sie als Allegorie des Bösen im Kampf mit dem personifizierten und läuternden Tod unterliegen lässt : das Signal für Biberkopfs Erlösung. Dieser Sieg des Todes wird durch ein fünfmal wiederholtes « O ja, o ja » bejubelt, ein Äquivalent für das « Halleluja! », mit dem in der "Offenbarung » der Fall Babylons begrüßt wird (offb. 18, 19). Symptomatisch ist, dass der Tod hier der Sieger ist und nicht Christus wie in der Bibel. So weit war Döblin 1929 offensichtlich noch nicht.

31 Zwar wird in "Berlin Alexanderplatz » wie im Neuen Testament die reinigende Kraft des Leidens und des Todes, der als "das wahre Leben» erscheint, hervorgehoben. Meiner Meinung nach ist es aber verfehlt, Biberkopf mit Christus gleichzusetzen, wie 
u.a. R.W. Fassbinder es 1980 tut, in seiner sonst sehr einfühlsamen und kongenialen Verfilmung.

Obschon die Faszination, die der Gekreuzigte auf den Romancier ausübte, bereits Mitte der zwanziger Jahre in Reise in Polen (1925/26) unübersehbar ist, fällt ebenso auf, dass er ihn noch als «Schmerzensmann » und Inbegriff des Leids in der Welt sieht.

\section{Von den religiösen zu den politischen Implikationen}

Der Umfang und das Gewicht der biblischen Bezüge im Berliner Roman signalisieren eine stärkere Prägung des Autors durch das Jüdisch-Christliche in den späten zwanziger Jahren, lange vor der Bekehrung zum Katholizismus. Als Jude war Döblin mit dem Alten Testament vertraut, obwohl er der jüdischen Religion fern blieb, 1912 die jüdische Gemeinde sogar verlassen hatte und sich erst 1923, nach den NovemberPogromen, der jüdischen Sphäre wieder zuwandte. 1924, während seiner Reise in Polen lernte er die jüdische Religiosität, vor allem den Chassidismus, näher kennen, der auch das Abzielen auf sich selbst und den Hochmut verurteilt. Dort interessierte Döblin sich, wie Th. Isermann ausführt, vor allem für die Gemeinsamkeiten zwischen Juden- und Christentum ${ }^{13}$.

Aber sein Rückgriff auf die Bibel ist nicht ohne Ambivalenz. Im Alten und im Neuen Testament begegnet ihm zwar das Irrationale, das Wunderbare und Visionäre, für das er sich immer schon empfänglich zeigte, jedoch wird das Übernatürliche meistens verinnerlicht und verweltlicht: Das Biblische fungiert als Gleichnis für eine seelische Krise des Menschen, als Ventil für die existenziellen Probleme des Schriftstellers, insbesondere als Ausdruck seiner Auseinandersetzung mit dem Verhältnis des Einzelnen zur Gemeinschaft - stets verflochten mit dem Problem von Hybris und Demut. Es geht dem Autor hauptsächlich um den ethischen Impetus der alttestamentlichen Geschichten.

Die Auslegung des Bibeltextes steht sogar in engem Zusammenhang mit Döblins damaligen politischen Ansichten. Im Roman wie in seinem ungefähr zeitgleichen Essay Unser Dasein betont er, dass der Mensch sich der Welt öffnen soll, statt sich hochmütig abzukapseln, ohne aber auf sein kritisches Denken zu verzichten. Dies führt auf politischem Gebiet, wie das Finale von «Berlin Alexanderplatz » nahe legt, zu einer Verurteilung des politischen Desinteresses und der Verblendung durch extremistische Programme. In der Endphase der Weimarer Republik, in der Zeit der sozialen Not und der sich vermehrenden Straßenkämpfe zwischen Nazis und Roten, empfiehlt Döblin einen Mittelweg zwischen einer «sozialistisch» geprägten Solidarität und der kritischen Reflexion des sich seiner Verantwortlichkeit bewussten Einzelnen, eine Einstellung, die er auch 1930/31 in Wissen und Verändern verteidigen sollte. In dieser Hinsicht geben die Bibelparaphrasen zu verstehen, dass diese ausgewogene Position erst nach der "kathartische(n) Grenzerfahrung der "Ohnmacht" am Rande des Lebens $»^{14}$, erst nach Überwindung der Egozentrik gewonnen werden kann. 


\section{NOTES}

1. An erster Stelle ist hier Werner Stauffachers penetranter Aufsatz « Die Bibel als poetisches Bezugssystem. Zu A. Döblins ,Berlin Alexanderplatz' », in Sprachkunst (1977, 8, S. 35-40) zu nennen, der eine unerlässliche Grundlage für weitere Untersuchungen geschaffen hat und dem wir viele Anregungen verdanken. Wertvolle Ausführungen über diesen Aspekt von Döblins Werk enthalten auch die Monographien von Helmut Schwimmer, A. Döblin. "Berlin Alexanderplatz" (München, Oldenbourg Verlag, 1973), Thomas Isermann, Der Text und das Unsagbare. Studien zu Religionssuche und Werkpoetik bei Döblin (Idstein, Schulz-Kirchner, 1989) und Jürgen Blume, Die Lektüren des A. Döblin (Frankfurt/Bern/NewYork/Paris, P. Lang, 1991).

2. Siehe z. B das leicht modifizierte Zitat aus Luk. (15.7) « Ein reuiger Sünder ist besser als 999 Gerechte« (S. 196), das Biberkopfs Selbstzufriedenheit andeutet, als er meint, Reinhold vom Mädchenhandel geheilt zu haben. Alle Zitate aus Berlin Alexanderplatz sind der Ausgabe aus dem Jahre 1996 entnommen (Hg. W. Stauffacher, Zürich, Düsseldorf, Walter-Verlag).

3. So lässt u.a. am Anfang des Romans eine unerwartet eingeschobene Anspielung auf Mordechai und Esther (S. 19 ; nach Buch Esther 2.5) die Erzieherrolle des alten Rebben für den verstörten Biberkopf ahnen.

4. Alfred Döblin : «Der Bau des epischen Werks », in Alfred Döblin : Aufsätze zur Literatur, Olten/ Freiburg, Walter-Verlag, 1963, S.106.

5. Werner Stauffacher : « Nachwort», in : A. Döblin, Berlin Alexanderplatz, S.840.

6. Siehe Werner Stauffacher, A.a.O., S. 40.

7. Siehe diesbezüglich Thomas Isermann, A.a.O., S.171 f.

8. S. $143 \mathrm{ff}$. Im vorletzten Buch des Romans, nach Miezes Ermordung, dem schwersten Schlag gegen Biberkopf, kommt der Schriftsteller nochmals auf die Parallelisierung mit Hiob zurück, in einer kurzen Paraphrase der Hiob-Geschichte nach K.1.15-20 ; K.2.7 f, 11 f (S. 379 f).

9. Robert Couffignal weist diesbezüglich auf eine gewisse Ähnlichkeit mit Johann Kaspar Lavaters Drama Abraham und Isaak. Ein religiöses Drama (1776) hin, das auch das Bibelgeschehen psychologisiert und sich einer gefühlsbetonten, sogar empfindsamen Sprache bedient. Robert Couffignal : L'Épreuve d'Abraham. Le récit de la Genèse et sa fortune littéraire, Toulouse, Université de Toulouse-Le Mirail, 1976, S. 82.

10. Herman Meyer : Das Zitat in der Erzählkunst, Stuttgart, Metzler, 1961, S. 12.

11. Siehe Thomas Isermann, A.a.O., S. 155.

12. Fragmente aus dieser Klage werden als Überschriften der Abschnitte eingesetzt, die von Biberkopfs Hilflosigkeit nach Miezes Verschwinden berichten (S. 379, 384, 386).

13. Thomas Isermann : A.a.O., S. 129.

14. Hans-Peter Bayerdörfer: "Der Wissende und die Gewalt. A. Döblins Theorie des epischen Werkes und der Schluss von "Berlin Alexanderplatz" », in : Matthias Prangel (Hg.), Materialien zu A. Döblin « Berlin Alexanderplatz », Frankfurt, Suhrkamp, 1975, S. 173.

\section{RÉSUMÉS}

Die Passagen aus dem Alten Testament und aus der Offenbarung Johannis, die Döblin in seinen 1929 erschienenen Großstadtroman einfügt, sind im Rahmen seiner Poetik und der von ihm 
befürworteten Erneuerung des Romans im Sinne der Moderne zu situieren. Die strukturierende und sinndeutende Funktion der eingeblendeten Bibelstellen wird durch die Analyse der einmontierten Hiob-Erzählung und der Abraham- Isaak-Geschichte verdeutlicht; andrerseits dokumentiert die Untersuchung der Variationen des Adam-und-Eva-Motivs aus der Genesis die mit den Bibelbezügen verbundene Potenzierung des dichterischen Reizes. Außerdem verleihen die eingeschobenen Sprüche Jeremias und des Predigers Salomo sowie die Auszüge aus der Offenbarung Johannis dem Berliner Roman eine ethische und metaphysische Dimension. Sie erscheinen als indirekte Kommentare des Erzählers, als lenkende Eingriffe des Autors in die Romanwelt. Der sehr freie Umgang mit der Vorlage, die vielen inhaltlichen und formalen Abweichungen vom Original signalisieren eine Säkularisierung und Humanisierung der biblischen Botschaft. Es geht hier nicht um die Unterwerfung unter eine Transzendenz, sondern um eine Reflexion über die Conditio humana und über das Verhältnis zwischen dem Einzelnen und dem Kollektiv, deren politische Implikationen nicht ubersehen werden sollten.

L'article analyse les modalités et la signification de l'intégration de passages de l'Ancien Testament et de l'Apocalypse dans ce roman polyphonique de 1929, célèbre pour sa «technique de montage ». Les emprunts à la Bible se situent dans le cadre du renouvellement de l'écriture romanesque réclamé par Doblin et sont traités dans un esprit moderniste (cf. J. Joyce, Th. Mann). La fonction principale de ces emprunts, la mise en évidence de la signification des personnages et de leur vécu, est étudiée à la lumière des extraits du Livre de Job et de l'histoire du sacrifice d'Abraham repris dans le roman. Les citations de versets du prophète Jérémie, de l'Ecclésiaste ainsi que de l'Apocalypse confèrent au récit, centré sur les bas-fonds de l'Est berlinois dans les années vingt, une dimension éthique, voire métaphysique, et sont assimilables à des commentaires indirects du narrateur, à des interventions normatives de l'auteur. Les libertés que prend Doblin vis-à-vis de l'original sont significatives. On constate une sécularisation et une humanisation du message biblique: il ne s'agit pas de soumission à une transcendance mais plutôt d'une réflexion sur la condition humaine et sur les rapports de l'individu à la collectivité, dont les implications politiques ne sont pas absentes. Sur le plan formel, l'auteur opte pour une langue concrète et ludique, pour le ton de la littérature populaire.

\section{AUTEUR}

\section{MONIQUE BOUSSART}

Université Libre de Bruxelles 